\title{
Pruning length of lateral branches influences auxin and cytokinins homeostasis in relation to growth and yields in Solanum lycopersicum L.
}

\section{Yang Xu}

Shandong Agricultural University

Xuelian Liu

Shandong Agricultural University

Chaoheng Gu

Shandong Agricultural University

Yanyan Yan

Shandong Agricultural University

Kai Li

Shandong Agricultural University

Qinghua Shi

Shandong Agricultural University

Fei Cheng

Qingdao Agricultural University

Lili Zhang

Shandong Province Seeds Group Co. Ltd

Changyong Shao

Shandong Province Seeds Group Co., Ltd

Biao Gong ( $\sim$ gongbiao@sdau.edu.cn )

Shandong Agricultural University https://orcid.org/0000-0003-3700-5967

\section{Research article}

Keywords: Auxin, Cytokinins, Lateral branches, Pruning, Tomato

Posted Date: December 5th, 2019

DOI: https://doi.org/10.21203/rs.2.18304/v1

License: (1) (1) This work is licensed under a Creative Commons Attribution 4.0 International License.

Read Full License 


\section{Abstract}

Lateral branches (LBs) pruning is performed frequently to keep the tomato plants in optimal growth conditions. However, the suitable pruning length of LBs, as well as it-mediated physiological and molecular mechanisms on plant growth regulation remains elusive in tomato. The effects of pruning length of LBs from 0 to $20 \mathrm{~cm}$ on vegetative growth, reproductive growth, labor costs, hormone metabolism and genes transcripts were evaluated on indeterminate type tomato cultivar. By comprehensive analysis, we provided evidence that pruning length of LBs at about $6 \sim 7 \mathrm{~cm}$ was suitable for plant growth, high yield, and low labor costs in tomato production. For mechanisms, appropriate extension of pruning length of LBs increased indole acetic acid (IAA) concentrations in root, which promoted the biosynthesis and upward transport of inactive cytokinins (CKs), as well as root development. Meanwhile, existence of LBs had inhibited effect on auxin outflow of the lower fruit stalks by testing transcripts of AUX1 and PIN, which increased the IAA and CKs concentrations of ovary and then promoted fruit setting and swelling. Additionally, pruning length of LBs also influenced the leaf senescence to control assimilation. Taken together, we highlighted that pruning length of LBs influenced auxin and cytokinins homeostasis in relation to growth and yields in tomato plants.

\section{Background}

Shoot branching is an important agronomic trait that directly determines plant architecture and affects plant growth and crop productivity (Barbier et al., 2019). During shoot branching, the axillary meristem in the leaf axils is activated, leading to generation of a lateral branch. Tomato (Solanum lycopersicum L.), a worldwide cultivated vegetable crop that is consumed freshly or processed into ketchup, has sympodial shoot architecture with determinate or indeterminate growth habits as well as bushy lateral branches phenotype (New et al., 2015). One-stem pruning approach is usually introduced in the current tomato production, especially for fresh market. Excess of lateral branches (LBs) often compete for nutrient allocation and light harvesting, and lead to disease especially in lower leaves, which generally have negative influences on tomato yield (Navarrete et al., 2000; Gong et al., 2019). Accordingly, though tomato pruning is labor intensive and time-consuming, LBs should be manually removed for maintaining a proper plant community and successful fruiting.

There is a general phenomenon that the growing shoot apex inhibits the outgrowth of LBs in plants, which is termed 'apical dominance' (Müller \& Leyser, 2011). Pruning of apical or lateral buds to control apical dominance has been widely used in horticultural production, which can balance vegetative growth and reproductive growth and create high-yield architecture for economic benefits. Auxin is known as the most direct and important signal molecule for apical dominance (Barbier et al., 2019). It also controls other aspects of developmental processes at the whole plant level, including lateral root formation, tropic responses, vascular initiation and differentiation, embryogenesis, and fruit development (El-Sharkawy et al., 2016). It is known that auxin is mainly synthesized in the young leaves of apical and lateral buds and subsequently transported basipetally in the polar auxin transport stream (PATS) based on auxin influx and efflux carriers (AUXs and PINs) (Ljung et al., 2001). Evidence shows that shoot-derived auxin is 
downward transported to roots and accounted as the major source of auxin to drive root growth and development (Morffy et al., 2018). However, not only auxin but also cytokinins (CKs) play central roles to control the development and architecture of the root system (Tang et al., 2017; Jing \& Strader, 2019). CKs antagonize auxin signaling by promoting the degradation of PIN1 protein (Marhavý et al., 2011) and interfering with PATS during lateral root organogenesis (Marhavý et al., 2014). Moreover, CKs have also been implicated as the second messenger of auxin for apical dominance, but display an antagonistic relationship with each other (Zha et al., 2019). Roots are the main CKs-producing organ in plants, and CKs biosynthesized in the roots is transported into the shoot system via the xylem (Shimizu-Sato et al., 2009).

Excepting apical dominance and root development, auxin and CKs also play important roles in regulation of fruit set and development, which are focused by tomato producers. The fruit set and development is associated with increased concentrations of auxin and CKs in tomato plant (Mariotti et al., 2011). So, exogenous synthetic analogues of auxin and CKs, such as 2,4-dichlorophenoxyacetic acid (2,4-D), 1naphthylacetic acid (NAA), 6-benzylaminopurine (6-BA), and $N$-(2-chloro-pyridin-4-yl)- $N^{\prime}$-phenylurea (CPPU), have been successfully and widely used in improving fruit set and development in fruit crops such as tomato, watermelon, melon, eggplant, grape and apple (Matsuo et al., 2012; Ding et al., 2013). Genetic evidences indicate that overexpression of auxin receptor TIR1 results in a positive effect on tomato fruits development (Ren et al., 2011; El-Sharkawy et al., 2016). Meanwhile, improving rootsynthesized CKs by overexpression of IPT can significantly enhance the tomato fruit yield, especially in stress condition (Ghanem et al., 2011). Until now, several auxin and CKs signaling participators, including auxin/IAA repressors (IAAs), auxin response factors ( $A R F S$ ) and cyclins (CYCA3;1 and $C Y C D 3 ; 1)$, have been identified and evidenced to influence the tomato fruit development (Joubès et al., 2000; de Jong et al., 2011; Su et al., 2014; Liu et al., 2018; Yuan et al., 2018).

Leaf senescence, a key high-yield constraint in later stage of tomato production, has also been linked with homeostasis of auxin and CKs (Gan \& Amasino, 2010; Mueller-Roeber \& Balazadeh, 2014). Overexpression of auxin- or CKs-synthesized gene ( $F Z Y$ or IPT) has been reported to delay leaf senescence (Ghanem et al., 2011; Kim et al., 2011). Similarly, multiple studies indicate that elevated levels of auxin and CKs cause the delay of leaf senescence (Gan \& Amasino, 2010; Mueller-Roeber \& Balazadeh, 2014). Moreover, loss-of-function Arabidopsis mutants (ahk2, ahk3 and ahk4) of CKs receptors show a senescence-sensitive phenotype. The above physiological and genetic evidences indicate that these two hormones play positive roles against leaf senescence.

In greenhouse tomato production, labor costs amount to about $30 \%$ to $50 \%$ of production costs. As competition among production areas throughout the world is increasing and tomato prices are decreasing, growers are trying to reduce production costs, in particular labor costs. As we known, there is often an inverse correlation between pruning length of LBs and labor intensity. Based on previous studies, we suppose that pruning length of LBs could change the homeostasis of auxin and CKs to influence the whole plant growth and development. Nevertheless, direct experimental evidence about this scientific problem remains elusive. Therefore, the present study is undertaken to investigate the effect as well as 
regulatory mechanism of pruning length of LBs on better growth of plant, higher yield, and low labor intensity in tomato production.

\section{Results}

\section{Influences of pruning length of LBs on plant growth}

We first compared the growth parameters of plant height and stem diameter when tomato plants were grown for 30, 60, 90 and $120 \mathrm{~d}$. No significant differences of these two parameters could be observed at $30 \mathrm{~d}$ after planting (Fig. 1A,B). However, values of plant height were increased with elevated pruning length of LBs, which peaked at T4, and then decreased from T5 to T7 at 60 and $90 \mathrm{~d}$ after planting (Fig. $1 \mathrm{~A})$. The plant heights of $\mathrm{T} 2$ to $\mathrm{T} 5$ treatments arrived at $2.5 \mathrm{~m}$ with removed apical buds at $120 \mathrm{~d}$ after planting, so the plant heights of T2 to T5 treatments were equal (Fig. 1A). Values of stem diameter showed similar trends with plant height, whose max values were shown in T3 from 60 to $120 \mathrm{~d}$ (Fig. 1B). The biomass accumulations showed that both root and shoot dry weight increased first and then decreased from $\mathrm{T} 1$ to $\mathrm{T} 7$ treatments, among which the max values were also observed in T4 treatments (Fig. 1C,D). However, enhanced ratios of root / shoot were shown with increased pruning length of LBs (Fig. 1E). This suggested an apparent interaction between plant growth and pruning length of LBs on plant physiology.

\section{Influences of pruning length of LBs on yields}

Early product launch usually brings an amount of economic benefits for growers, especially for inverseseasonal vegetable production in greenhouse. Excitedly, fruit weight of the first inflorescence showed that the early tomato yield was increased with the increased pruning length of LBs (Fig. 2A). However, the pruning lengths of LBs in T5 to T7 treatments were not always benefited for fruit yield with the growth of tomato plants as well as fruit setting. The fruit weights of the second to fifth inflorescences increased first and then decreased from T1 to T7 treatments, among which the max yield was obtained in T4 treatments (Fig. 2A). The best value for total yield was recorded by the T4 treatment that was $15.2 \%$ and $12.8 \%$ higher as compared to T1 and T7 treatments, respectively (Fig. 2B). Accordingly, it is highlighted that pruning length of LBs played a critical role in regulating vegetative growth and reproductive growth to influence tomato yield.

\section{Influences of pruning length of LBs on labor costs}


Labor costs were estimated by two kinds of measurements: numbers of removed branches and pruning times. As shown in Fig. 3, numbers of both removed branches and pruning times were all decreased with the increased pruning length of LBs. It should be noted that T4 treatment not only improved the plant growth and yield but also decreased the numbers of removed branches and pruning times by $45.9 \%$ and $52.3 \%$ when compared with T1 treatment (Fig. 3). Taken together, Fig. 2 and 3 showed that appropriate extension of the pruning length of LBs to $6 \sim 7 \mathrm{~cm}$ could improve tomato yield and save labor costs when compared with traditional pruning approach of $\mathrm{T} 1$ treatment. According to a comprehensive estimation for the above parameters, treatments of $\mathrm{T} 1, \mathrm{~T} 4$ and $\mathrm{T} 7$ were used for further studies to unravel the regulatory mechanism of pruning length of LBs on the reproductive/vegetative balance on the tomato plant.

\section{Influences of pruning length of LBs on auxin and CKs signaling in roots}

Current opinion indicates that auxin promotes but CKs inhibits root growth and development (Marhavý et al., 2011; Marhavý et al., 2014; Morffy et al., 2018; Jing \& Strader, 2019). Root IAA concentrations of T4 and T7 treatments were about $61.6 \%$ and $121.1 \%$ higher than that of T1 treatment (Fig. 4A). Meanwhile, T4 and T7 treatments showed significantly lower CKs accumulation involved both active forms (IP, tZ and cZ) and storage forms (DZ) of CKs in roots (Fig. 4B). However, the differences of tZ, cZ and DZ forms in T4 and T7 treatments were not significant (Fig. 4B). In comparison, T7 had a significantly inhibiting effect on IP forms accumulation when compared with T4 treatment (Fig. 4B). Thus, we confirmed that pruning length of LBs had significant effects on auxin and CKs homeostasis in roots.

Though there were significant differences of IAA concentrations among T1, T4 and T7, no differences of IAA biosynthesis gene (flavin monooxygenase, FZY1) transcript were detected in these treatments (Fig. 4C). For auxin signaling, down-regulated transcript of IAA16 with up-regulated transcripts of $A R F 5$ and ARF7 were shown in T4 and T7 treatments when compared with $\mathrm{T} 1$ treatment (Fig. 4C). These results implied that though the enhanced levels of IAA were not de novo synthesized in roots of T4 and T7 treatments, which could activate the auxin signaling in tomato roots. Meanwhile, transcripts of genes involving CKs synthesis (adenosine phosphate-isopentenyltransferase, IPT2) and degradation (cytokinin oxidase, CKX2) were up-regulated, but CKs activation (LONELY GUY, LOG2) and signaling (type-A response regulators, $T R R 3 / 4$ and $T R R 7 / 15$ ) were down-regulated in $\mathrm{T} 4$ and $\mathrm{T} 7$ treatments when compared with T1 treatment (Fig. 4C).

\section{Influences of pruning length of LBs on auxin and CKs signaling in fruits}


Because auxin plays the critical role in fruit development, we first addressed the involvement of $A U X 1$ and PIN1 in mediating the auxin flow from the fruit stalks. Both transcripts of AUX1 and PIN1 were significantly up-regulated after pruning treatment (Fig. 5A,B), which indicated that the upper LBs had inhibited effects on auxin outflow of the lower fruit stalks. Importantly, the transcripts of AUX1 and PIN1 showed rising abundance with increased length of LBs before pruning treatment, but interestingly, no significant differences were observed among $\mathrm{T} 1, \mathrm{~T} 4$ and $\mathrm{T} 7$ treatments after pruning treatment (Fig. $5 A, B)$.

Correlating with the establishment of auxin export by $A U X 1$ and PIN1 from the fruit stalks, a significantly rising gradient of IAA was shown from T1 to T7 in ovaries (Fig. 5C). For CKs homeostasis of ovaries, T4 and T7 treatments showed significantly higher accumulations of four types of CKs when compared with T1 treatment; however, little difference was shown between each other (Fig. 5D).

The transcripts of $F Z Y 1, A R F 5$ and $A R F 7$ were up-regulated, but IAA14 transcript was down-regulated in T4 and T7 treatments when compared with T1 treatment (Fig. 5E), which is correlative with IAA homeostasis (Fig. 5C). Meanwhile, transcripts of IPT2, LOG2, LOG6 and CYCA3; 7 were up-regulated, but CKX2 transcript was down-regulated in T4 and T7 treatments when compared with T1 treatment (Fig. 5E), which is correlative with CKs homeostasis (Fig. 5D).

\section{Influences of pruning length of LBs on auxin and CKs signaling in lower leaves}

Leaf senescence is a form of programmed cell death and is characterized by decline in both chlorophyll and photosynthesis (Dai and Dong, 2011). The max values of chlorophyll concentrations, $F_{v} / F_{m}$ and $\mathrm{P}_{\mathrm{N}}$ were shown in T4 treatment, and the subsequent values were shown in T7 treatment (Fig. 6A-C), which indicated that suitably increasing the pruning length of LBs was benefit for delaying senescence of the lower leaves. To analyze the underlying molecular mechanisms for senescence regulation by pruning length of LBs, we examined the transcripts of four important senescence-responsive genes. One senescence-resistive gene of CRF6 and three senescence-sensitive genes of MYB2, SAG12 and ORE1 showed positive and negative correlations separately with the above senescence-related indicators (Fig. $6 \mathrm{~F}$ ). Importantly, both concentrations of auxin and CKs showed similar trends with the above senescencerelated indicators (Fig. 6D,E). However, no significant differences were observed in transcripts of auxinand CKs-synthetic genes (FZY1 and IPT2) in different treatments (Fig. 6F). Interestingly, reduced transcripts of PIN1 and $C K X 2$, as well as enhanced transcripts of LOG2 were shown from T1 to T7 treatments (Fig. 6F). Taken together, these results indicated that the homeostasis of these two hormones were mainly regulated by auxin flow and CKs activation and degradation through changing pruning length of LBs, which further influenced the senescence-related gene expression as well as senescence process of lower leaves. 


\section{Discussion}

The present study highlighted that LBs of tomato plants should be removed at a suitable length at about $6 \sim 7 \mathrm{~cm}$ (T4 treatment), which was benefit for promoting plant growth and yield, as well as for reducing labor costs when compared with blindly or lazily pruning (T1 or T7 treatments). Considering that auxin is mainly synthesized in the young leaves of apical and lateral buds (Ljung et al., 2001). We thought that pruning length of LBs could change the auxin homeostasis, which combined with CKs to play important roles in regulating the whole plant growth and development. However, the physiological and molecular mechanisms of the regulation of pruning length of LBs on plant growth and yield have not been well investigated in fruit crops. The present study, as far as is known for the first time, described a complete set of auxin and CKs homeostasis and signaling in responding to pruning length of LBs for revealing the mechanisms of pruning-regulated plant growth and yield at hormone levels in tomato plants.

One of the main functions of roots is in the acquisition of water and mineral nutrients from the soil environment (Shin et al., 2007). To maximize the capture of nutrients utilization, roots may proliferate or elongate resulting in increased root weight and root / shoot ratio for the patches of soil that contain mineral nutrients. Here, we found that tomato plants with pruning length of LBs at about $6 \sim 7 \mathrm{~cm}$ (T4 treatment) had an optimal architecture for high yield production as indicated by the increased root dry weight, shoot dry weight and root / shoot ratio (Fig. 1). As we known, lateral roots constituted the major components of the whole root system for both biomass and function. Previous studies indicated that interplay of auxin and CKs played central roles during lateral root development (Jing and Strader, 2019). Decades ago, it was described that auxin promoted but CKs inhibited the lateral root formation (Bottger, 1974; Goodwin and Morris, 1979; Wightman, et al., 1980). Subsequent studies indicate that cytokinin treatment inhibits lateral root initiation and development, converse to the stimulatory effects of auxin treatment (Mason et al., 2005; Li et al., 2006). In accordance with root dry weight (Fig. 1C), significantly enhanced concentrations of IAA and reduced concentrations of four forms of CKs were observed in T4 treatment when compared with $\mathrm{T} 1$ treatment (Fig. $4 \mathrm{~A}, \mathrm{~B})$. Because there was no difference of $F Z Y 1$ expression among T1, T4 and T7 treatments, we considered that the enhanced accumulation of IAA was mainly obtained from the LBs of shoot. The gain-of-function mutation of IAA16 has been reported to display resistance to the auxin and impede root elongation and fertility in Arabidopsis (Rinaldi et al., 2012). Additionally, loss function of $A R F 5$ and $A R F 7$ inhibited lateral root development and showed a SOLITARY ROOT phenotype in Arabidopsis (Okushima et al., 2005; Smet, 2010). Thus, the reduced transcripts of IAA16 and enhanced transcripts of ARF5 and ARF7 in T4 treatment were responsible for the positive auxin signaling response and root development (Fig. $1 \mathrm{C}$ and $4 \mathrm{C}$ ). Auxin has been found to stimulate oxidative breakdown of active CKs, which is mediated by CKXs (Coenen and Lomax, 1997). The varying patterns of IPT2, LOG2 and CKX2 (Fig. 4C) implied that enhanced accumulations of IAA in T4 and T7 treatments resulting in total CKs biosynthesis; however, the active CKs were degraded by $C K X 2$ or inactivated by $L O G 2$ in roots. To our interesting, both active and inactive forms of CKs were reduced in T4 and T7 treatments (Fig. 4B), which implied that the inactive CKs might be transported and activated in shoot. Moreover, auxin has also been demonstrated to have effects on increasing the activity of CKX at enzyme levels (Palni et al., 1998). So, we also examined the expression of TRRs, which have been used 
as markers of CKs signaling (Shani et al., 2010). The transcripts of TRR3/4 and TTR7/15 were decreased with the increased pruning length of LBs (Fig. 4C). Together with changes in CKs concentrations, these results suggest that the CK signal transduction pathway is partly inhibited by the excess LBs. Totally, these evidences suggested that adequately increasing the pruning length of LBs has a positive effect on root development via regulating the hormones and signaling of auxin and CKs.

The competition between vegetative growth and reproductive growth is universal law in plants, especially in fruit crops (Navarrete and Jeannequin, 2000). The vegetative growth of tomato is estimated visually by measurements of stem diameter and shoot dry weight. Both notations are useful: though the stem diameter is an objective indicator of the vegetative growth, the shoot dry weight is an accurate estimation of the vegetative growth and is more comprehensive than stem diameter (Hall, 1983; Navarrete et al., 1997). In this study, when pruning length of LBs reaches to $8 \sim 10 \mathrm{~cm}$, the stem diameter and shoot dry weight are significantly lower in comparison with the pruning length of LBs at $4 \sim 7 \mathrm{~cm}$ (Fig. 1B,C), which indicates a decrease in vegetative growth (Navarrete and Jeannequin, 2000). The phenomenon of deficient vegetative growth in T5 T7 treatments led to a significant decrease in total yield (Fig. 2B) and in particular in later yield of the fourth and fifth inflorescences (Fig. 2A). Hartmann (1977) showed that non pruning operation reduced the tomato yield liking our result of T8 treatment: one explanation is that instead of assigning part of assimilates to tomato fruit, large part of assimilates is diverted to the LBs. In T1 and T2 treatments, the LBs are pruned frequently. Therefore, the labor costs are significantly higher than other treatments (Fig. 3). In this case, the stem diameter, shoot dry weight and yield are significantly lower than that in T3 and T4 treatments (Fig. 1B,D and Fig. 2B). We consider that this phenomenon is mainly caused by three reasons. First, weakly root system has incompetent nutrition and water support for both vegetative growth and reproductive growth (Fig. 1C). Second, deficits of LBs-sourced auxin and root-sourced CKs (Fig. 4A,B; Fig. 5C,D and Fig. 6D,E) have detrimental effects on cell differentiation and elongation at the whole plants level (Su et al., 2011). Third, the mechanical stress, which is caused by the high frequency of pruning operations, is also considered as a inhibited factor for vegetative growth and reproductive growth. Several lines of evidence indicate that most of the mechanical stresses tested reduce leaf growth, stem diameter and even sometimes yield on several crop materials, which is especially serious on tomato plants (Heuchert and Mitchell, 1983; Biddington, 1986; Mitchell and Myers, 1995). Taken together, These explain why T4 treatment show better effects than T1 and T7 treatments for pruning management in tomato production.

Except for the balance between vegetative growth and reproductive growth, hormones of auxin and CKs also play important roles in fruit setting and swelling and then influence the tomato yield. The homeostasis of PATS is important for fruit retention and development (Pattison and Catalá, 2012). Because excessive PATS could reduce the concentrations of auxin in ovaries, which is postulated to have a direct effect on assimilate partitioning (Agusti et al., 2002). If PATS is reduced too much, the fruit may abscind by the activation of an abscission zone at the base of the subtending organ (Else et al., 2004). The PATS velocity is influenced most strongly by the activity of AUX1 and PIN1 (Pattison and Catalá, 2012). We found that pruning operations significantly enhanced the transcripts of $A U X 1$ and PIN1 (Fig. $5 A, B)$. Moreover, shorter pruning length of $L B s(T 1)$ in "before pruning" treatment showed similar 
expression patterns of $A U X 1$ and PIN1 with that in "after pruning" treatment (Fig. 5A,B). These data suggest that adequate reserve LBs is important for PATS homeostasis, which further influence the fruit yield. In recent years, the molecular mechanisms of the auxin and CKs signaling cascades have been well characterized in tomato fruit setting (Else et al., 2004; de Jong et al., 2011; Ding et al., 2013; El-Sharkawy et al., 2016). In tomato, both ARF5 (Liu et al., 2018) and ARF7 (de Jong et al., 2011) have been demonstrated to act as a negative regulator of fruit set. Furthermore, function of $A R F 7$ depends on interaction with IAA14 (Ito, J. et al. 2016). So, high transcripts of $A R F 5$ and ARF7 with low transcripts of IAA14 (Fig. 5E) can be responsible for yield inhibition that is caused by strong vegetative growth at hormone signaling levels. For CKs signaling, transcripts of IPT2, LOG2, LOG6 and CYCD3;1 showed positively response to CKs accumulation (Fig. 5D,E). Similar results can be observed in both exogenous CKs-treated or pollinated tomato fruits (Matsuo et al., 2012). The present study provides evidence that retention of LBs is benefit for maintaining the PATS of main stem, which inhibits the PATS of fruit stalks and promotes root development for CKs generation and ascending transport (Fig. 5). Thus, the transcripts of auxin- or CKs-related signaling genes were activated during early fruit development (Fig. 5E).

A unsuspected phenotype of retardant senescence of tomato lower leaves was observed in T4 treatment when compared with $\mathrm{T} 1$ and $\mathrm{T} 7$ treatments (Fig. 6A-C). The transcripts of $F Z Y 1$ and PIN1 imply that the influence of pruning length of LBs on the IAA concentrations of lower leaves remain depends on PATS (Fig. 6E,F). Thus, high level of IAA can be responsible for dominant nutrients competition, which delays senescence (Agusti et al., 2002). Previous study showed that using constitutive IPT-expressing rootstock (35S::IPT) significantly enhanced the shoot CKs levels and inhibited the leaf senescence in salinized tomato plants (Ghanem et al., 2011). And we also found that the concentrations of CKs were significantly higher in T4 treatment when compared with T1 and T7 treatments (Fig. 6E). Meanwhile, the transcript patterns of IPT2, LOG2 and CKX2 indicated that the enhanced CKs mainly synthesized in root and activated in leaves (Fig. 6E,F). These results are compatible with well developed root system in T4 treatment (Fig. 1C). Together with IAA, CKs play key roles in regulating senescence-related genes expression of CRF6, MYB2, SAG12 and ORE1 against senescence (Fig. 6F). Based on hormone theory, we have discussed the different senescence phenotype between T1 and T4 treatment. When compared with T4 treatment, T7 treatment significantly increased lower leaves density, which led to less sunlight and senescence phenotype. Thus, the stronger photosynthesis of lower leaves in T4 treatment can also contribute assimilation for high fruit yield.

\section{Conclusion}

The present study demonstrates that pruning length of LBs plays important roles in regulating whole plant growth and development, as well as fruit yield in tomato plants. As shown in Fig. 7, the crucially contributory factor is that LBs are IAA sources, which can influence the PATS by pruning operation. Changed PATS regulates root IAA concentrations to promote or inhibit root development, which is the main sources of CKs to control fruit setting and leaf senescence. Thus, the pruning-induced homeostasis of auxin and CKs are comprehensively influence the whole plant growth and development. At last, we 
highlight that pruning length of LBs at about $6 \sim 7 \mathrm{~cm}$ is suitable for plant growth, high yield, and low labor costs in tomato production.

\section{Methods}

\section{Cropping conditions, plant material and experimental design}

This experiment was conducted in a solar greenhouse located at Shandong Agricultural University, Tai'an, China $\left(117^{\circ} \mathrm{E}\right.$ and $\left.36^{\circ} \mathrm{N}\right)$. The mean temperatures were $18^{\circ} \mathrm{C}$ at night and $28^{\circ} \mathrm{C}$ at day in spring and autumn, and $10{ }^{\circ} \mathrm{C}$ at night and $25^{\circ} \mathrm{C}$ at day in winter.

Seeds of tomato (Solanum lycopersicum L.) cultivar 'Qidali' (Syngenta; acquired from the Shandong Province Seeds Group Co., Ltd, Jinan 250100, P.R. China) were sown in $50 \mathrm{ml}$ plastic pot filled with a mixture of peat and vermiculite. When seedlings had 4 true leaves, they were planted onto field in the greenhouse. One-stem pruning approach was conducted. And the trusses were pruned to five inflorescences with four fruits in each inflorescence. The apical buds were removed when plant height arrived at $2.5 \mathrm{~m}$. The plants were grown in the greenhouse until the harvest stopped.

This experiment included seven treatments depending on the pruning length of LBs: T1 $(0 \sim 1 \mathrm{~cm})$, T2 $(2$ $\sim 3 \mathrm{~cm})$, T3 $(4 \sim 5 \mathrm{~cm})$, T4 $(6 \sim 7 \mathrm{~cm})$, T5 $(8 \sim 10 \mathrm{~cm})$, T6 $(10 \sim 15 \mathrm{~cm})$ and T7 $(15 \sim 20 \mathrm{~cm})$. The experiment was set up in a randomized complete block design with three replicates, and each replicate contained twenty plants.

\section{Parameters of plant growth, yield and labor costs}

Plant height and stem diameter were measured at 30,60, 90, $120 \mathrm{~d}$ after planting. Roots and shoots were collected at $120 \mathrm{~d}$ after planting and then oven-dried to get root and shoot dry weight. Fruit weight of each inflorescence and total yield were measured when the fruit ripening. Numbers of removed branches and pruning times were recorded for labor costs evaluation.

\section{RNA extraction and gene expression analysis}

Total RNA was extracted from roots, ovaries and leaves using TRIzol method (Gong et al., 2014). Then, cDNA synthesis was performed according to standard procedures of a SuperScript ${ }^{\mathrm{TM}}$ First-Strand Synthesis System (Invitrogen). Three technical replicates of qRT-PCR were performed per primer pair (Supplementary Table 1) using an aliquot of cDNA (1/500), Power SYBR Green PCR Master Mix (ABI), 
and $200 \mathrm{nM}$ each primer on an ABI Prism $7900 \mathrm{HT}$ machine. Relative expression was calculated according to Vandesompele et al. (2002) and Yan et al. (2019)

\section{Measurements of endogenous phytohormones}

Concentrations of indole acetic acid (IAA) and CKs (N6-isopentenyladenine, IP; trans-Zeatin, tZ; cis-Zeatin, cZ; Dihydrozeatin, DZ) were detected by liquid chromatography-tandem mass spectrometry methods

(Roman et al., 2016). About $20 \mathrm{mg}$ samples of roots, ovaries and leaves were homogenized and extracted in $1 \mathrm{~mL}$ Bieleski buffer (methanol : methanoic acid : water $=6: 1: 3, \mathrm{~m}: \mathrm{m}: \mathrm{m}$ ) together with a cocktail of stable isotope-labeled internal standards $(0.25 \mathrm{pmol}$ of CKs bases, ribosides, and N-glucosides, $0.5 \mathrm{pmol}$ of CKs O-glucosides and nucleotides, and $5 \mathrm{pmol}$ of $\left[{ }^{2} \mathrm{H}_{5}\right]$ IAA per sample added). The extracts were purified using two solid-phase extraction columns, the octadecylsilica-based column (C18, $500 \mathrm{mg}$ of sorbent; Applied Separations) and after that the Oasis MCX column (30 $\mathrm{mg} \mathrm{mL}^{-1}$; Waters). Analytes were eluted by a three step elution using a $60 \%(\mathrm{v} / \mathrm{v})$ methanol, $0.35 \mathrm{M} \mathrm{NH}_{4} \mathrm{OH}$ aqueous solution and $0.35 \mathrm{M}$ $\mathrm{NH}_{4} \mathrm{OH}$ in $60 \%(\mathrm{v} / \mathrm{v})$ methanol solution. IAA and CKs levels were determined using ultra-HPLCelectrospray-tandem mass spectrometry using stable isotope-labeled internal standards as a reference.

\section{Statistical Analysis}

ANOVA statistical analyses were performed on all data using SPSS $15.0(P<0.01)$ and data were tested for significant differences $(P<0.05)$ using Tukey's test. Means and standard errors were calculated from three biological replicates.

\section{Declarations}

Ethics approval and consent to participate: Not applicable.

Consent for publication: Not applicable.

Availability of data and material: The materials used during the current study will be freely available upon request to corresponding author: gongbiao@sdau.edu.cn.

Competing interests: The authors declare that they have no competing interests.

Funding: The National Natural Science Foundation of China [31872943]; the Shandong Provincial Natural Science Foundation, China [ZR2019MC067]; the Shandong Province Modern Agricultural Technology System [SDAIT-05-05]. The founders did not play any roles in the design of the study; collection, analysis, and interpretation of data; and in writing the manuscript. 
Authors' contributions: $X Y$ and $B G$ conceived of and designed the study. $X Y, X L$ and $C G$ conducted the experiments. $\mathrm{KL}$ and $\mathrm{YY}$ performed qRT-PCR analysis. FC, LZ and CS performed data analysis. BG wrote the manuscript. QS supervised and complemented the writing. All authors have read and approved this manuscript.

Acknowledgements: Not applicable.

\section{Abbreviations}

2,4-D: 2,4-dichlorophenoxyacetic acid; 6-BA: 6-benzylaminopurine; CKs: cytokinins; CPPU: $N$-(2-chloropyridin-4-yl)-N'phenylurea; cZ: cis-Zeatin; DZ: Dihydrozeatin; IAA: indole acetic acid; IP: N6isopentenyladenine; LBs: lateral branches; NAA: 1-naphthylacetic acid; PATS: polar auxin transport stream; tZ: trans-Zeatin.

\section{References}

1. Agusti, M., Zaragoza, S., Iglesias, D.J., Almela, V., Primo-Millo, E. \& Talon, M. The synthetic auxin 3, 5, 6-TPA stimulates carbohydrate accumulation and growth in citrus fruit. Plant Growth Regul. 36, 141147. (2002).

2. Barbier, F.F., Dun, E.A., Kerr, S.C., Chabikwa, T.G. \& Beveridge, C.A. An update on the signals controlling shoot branching. Trends Plant Sci. 24, (2019).

3. Biddington, N. The effect of mechanically induced stress in plants - a review. Plant Growth Regul. 4, 103-123. (1986).

4. Bottger, M. Apical dominance in roots of Pisum sativum Planta 121, 253-261. (1974).

5. Coenen, C. and Lomax, T.L. Auxin-cytokinin interactions in higher plants: old problems and new tools. Trends Plant Sci. 2, 351-356. (1997).

6. Dai, J. \& Dong, H. Stem girdling influences concentrations of endogenous cytokinins and abscisic acid in relation to leaf senescence in cotton. Acta Physiol. Plant 33, 1697-1705. (2011).

7. de Jong, M., Wolters-Arts, M., García-Martínez, J.L., Mariani, C. \& Vriezen, W.H. The Solanum lycopersicum AUXIN RESPONSE FACTOR 7 (SIARF7) mediates cross-talk between auxin and gibberellins signalling during tomato fruit set and development. Exp. Bot. 62, 617-626. (2011).

8. Ding, J., Chen, B., Xia, X., Mao, W., Shi, K., Zhou, Y. \& Yu, J. Cytokinin-induced parthenocarpic fruit development in tomato is partly dependent on enhanced gibberellin and auxin biosynthesis. Plos One 8, (2013).

9. El-Sharkawy, I., Sherif, S., Kayal, W.E., Jones, B., Li, Z., Sullivan, A.J. \& Jayasankar, S. Overexpression of plum auxin receptor PSITIR1 in tomato alters plant growth, fruit development and fruit shelf-life characteristics. BMC Plant Biol. 16, (2016).

10. Else, M.A., Stankiewicz-Davies, A.P. Crisp, C.M. \& Atkinson C.J. The role of polar auxin transport through pedicels of Prunus avium in relation to fruit development and retention. J. Exp. Bot. 55, 
2099-2109. (2004).

11. Gan, S. \& Amasino, R.M. Cytokinins in plant senescence: From spray and pray to clone and play. Bioessays 18, 557-565. (2010).

12. Ghanem, M.E., Albacete, A., Smigocki, A.C., Frébort, l., Pospíšilová, H., Martínez-Andújar, C., Acosta, M., Sánchez-Bravo, J., Lutts, S., Dodd, I.C. \& Pérez-Alfocea, F. Root-synthesized cytokinins improve shoot growth and fruit yield in salinized tomato (Solanum lycopersicum) plants. J. Exp. Bot. 62, 125-140. (2011).

13. Gong, B., Li, X., VandenLangenberg, K.M., Wen, D., Sun, S., Wei, M., Li, Y., Yang, F., Shi, Q. \& Wang. X. Overexpression of S-adenosyl-L-methionine synthetase increased tomato tolerance to alkali stress through polyamine metabolism. Plant Biotechnol. J. 12, 694-708. (2014).

14. Gong, B., Yan, Y., Zhang, L., Cheng, F., Liu, Z. \& Shi, Q. Unravelling GSNOR-mediated S-nitrosylation and multiple developmental programmes in tomato plants. Plant Cell Physiol. pcz143, https://doi.org/10.1093/pcp/pcz143 (2019).

15. Goodwin, P.B. \& Morris, S.C. Application of phytohormones to pea roots after removal of the apexeffect on lateral root production. J. Plant Physiol. 6, 195-200. (1979).

16. Hall, D. The influence of nitrogen concentration and salinity of recirculating solutions on the early season vigour and productivity of glasshouse tomatoes. Hort. Sci. 58, 411-415. (1983).

17. Hartmann, H.D. Influence of axillary shoots on growth and yield of tomato varieties. Gartenbauwissenschaft 42, 178-184. (1977).

18. Heuchert, J.C. \& Mitchell, C.A. Inhibition of shoot growth in greenhouse tomato by periodic gyratory shaking. Am. Soc. Hort. Sci. 108, 801-805. (1983).

19. Ito, J., Fukaki, H. Onoda, M., Li, L., Li, C., Tasaka, M. \& Furutani, M. Auxin-dependent compositional change in mediator in ARF7- and ARF19-mediated transcription. Natl. Acad. Sci. 113, 6562-6567. (2016).

20. Jing, H. \& Strader, L.C. Interplay of auxin and cytokinin in lateral root development. J. Mol. Sci. 20, 486. (2019).

21. Joubès, J., Walsh, D., Raymond, P. \& Chevalier, C. Molecular characterization of the expression of distinct classes of cyclins during the early development of tomato fruit. Planta 211, 430-439. (2000).

22. Kim, J.I., Murphy, A.S., Baek, D., Lee, S.W., Yun, D.J., Bressan, R.A. \& Narasimhan M.L. YUCCA6 overexpression demonstrates auxin function in delaying leaf senescence in Arabidopsis thaliana. Exp. Bot. 62, 3981-3992. (2011).

23. Li, X., Mo, X.R., Shou, H.X. \& Wu, P. Cytokinin-mediated cell cycling arrest of pericycle founder cells in lateral root initiation of Arabidopsis. Plant Cell Physiol. 47, 1112-1123. (2006).

24. Liu, S., Zhang, Y., Feng, Q., Qin, L., Pan, C., Lamin-Samu, A.T. \& Lu, G. Tomato AUXIN RESPONSE FACTOR 5 regulates fruit set and development via the mediation of auxin and gibberellin signaling. Rep-UK 8, 2971. (2018). 
25. Ljung, K., Bhalerao, R.P. \& Sandberg, G. Sites and homeostatic control of auxin biosynthesis in Arabidopsis during vegetative growth. Plant J. 28, 465-474. (2001).

26. Marhavý, P., Bielach, A., Abas, L., Abuzeineh, A., Duclercq, J., Tanaka, H., Pařezová, M., Petrášek, J., Friml, J., Kleine-Vehn, J. \& Benková, Cytokinin modulates endocytic trafficking of PIN1 auxin efflux carrier to control plant organogenesis. Dev. Cell 21, 796-804. (2011).

27. Marhavý, P., Duclercq, J., Weller, B., Feraru, E., Bielach, A., Offringa, R., Friml, J., Schwechheimer, C., Murphy, A. \& Benková, E. Cytokinin controls polarity of PIN1-dependent auxin transport during lateral root organogenesis. Biol. 24, 1031-1037. (2014).

28. Mariotti, L., Picciarelli, P., Lombardi, L. \& Ceccarelli, N. Fruit-set and early fruit growth in tomato are associated with increases in indoleacetic acid, cytokinin, and bioactive gibberellin contents. Plant Growth Regul. 30, 405-415. (2011).

29. Mason, M.G., Mathews, D.E., Argyros, D.A., Maxwell, B.B., Kieber, J.J., Alonso, J.M., Ecker, J.R. \& Schaller, G.E. Multiple type-B response regulators mediate cytokinin signal transduction in Arabidopsis. Plant Cell 17, 3007-3018. (2005).

30. Matsuo, S., Kikuchi, K., Fukuda, M., Honda, I. \& Imanishi, S. Roles and regulation of cytokinins in tomato fruit development. Exp. Bot. 63, 5569-5579. (2012).

31. Mitchell, C. \& Myers, P. Mechanical stress regulation of plant growth and development. Rev. 17, 1-41. (1995).

32. Morffy, N.J. \& Strader, L.C. Locally sourced: auxin biosynthesis and transport in the root meristem. Cell 47, 262-264. (2018).

33. Mueller-Roeber, B. \& Balazadeh, S. Auxin and its role in plant senescence. Plant Growth Regul. 33, 2133. (2014).

34. Müller, D. \& Leyser, O. Auxin, cytokinin and the control of shoot branching. Bot-London 107, 12031212. (2011).

35. Navarrete, M. \& Jeannequin, B. Effect of frequency of axillary bud pruning on vegetative growth and fruit yield in greenhouse tomato crops. Hortic-Amsterdam 86, 197-210. (2000).

36. Navarrete, M., Jeannequin, B. \& Sebillotte, M. Vigour of greenhouse tomato plants (Lycopersicon esculentum): analysis of the criteria used by growers and search for objective criteria. J. Hort. Sci. 72, 821-829. (1997).

37. New Lee, O., Uchida, Y., Nemoto, K., Mine, Y. \& Sugiyama N. Quantitative trait loci analysis of lateral shoot growth in tomato. Hortic-Amsterdam 192, 117-124. (2015).

38. Okushima, Y., Overvoorde, P.J., Arima, K., Alonso, J.M., Chan, A., Chang, C., Ecker, J.R., Hughes, B., Lui, A., Nguyen, D., Onodera, C., Quach, H., Smith, A., Yu, G. \& Theologisa, A. Functional genomic analysis of the AUXIN RESPONSE FACTOR gene family members in Arabidopsis thaliana: unique and overlapping functions of ARF7 and ARF19. Plant Cell 17, 444-463. (2005).

39. Palni, L.M.S., Burch, L. and Horgan, R. The effect of auxin concentration on cytokinin stability and metabolism. Planta 174, 231-234. (1988). 
40. Pattison, R.J. \& Catalá, C. Evaluating auxin distribution in tomato (Solanum lycopersicum) through an analysis of the PIN and AUX/LAX gene families. Plant J. 70, 585-598. (2012).

41. Ren, Z., Li, Z., Miao, Q., Yang, Y., Deng, W. \& Hao, Y. The auxin receptor homologue in Solanum lycopersicum stimulates tomato fruit set and leaf morphogenesis. Exp. Bot. 62, 2815-2826. (2011).

42. Rinaldi, M.A., Liu, J., Enders, T.A., Bartel, B. \& Strader, L.C. A gain-of-function mutation in IAA16 confers reduced responses to auxin and abscisic acid and impedes plant growth and fertility. Plant Mol. Biol. 79, 359-373. (2012).

43. Roman, H., Girault, T., Barbier, F., Péron, T., Brouard, N., Pěnčík, A., Novák, O., Vian, A., Sakr, S., Lothier, J., Gourrierec, J.L. \& Leduc, N. Cytokinins are initial targets of light in the control of bud outgrowth. Plant Physiol. 172, 489-509. (2016).

44. Shani, E., Ben-Gera, H., Shleizer-Burko, S., Burko, Y., Weiss, D. \& Ori, N. Cytokinin regulates compound leaf development in tomato. Plant Cel/ 22, 3206-3217. (2010).

45. Shimizu-Sato, S., Tanaka, M. \& Mori, H. Auxin-cytokinin interactions in the control of shoot branching. Plant Mol. Biol. 69, 429-435. (2009).

46. Shin, R., Burch, A.Y., Huppert, K.A., Tiwari, S.B., Murphy, A.S., Guilfoyle, T.J. \& Schachtman, D.P. The Arabidopsis transcription factor MYB77 modulates auxin signal transduction. Plant Cell 19, 24402453. (2007).

47. Smet, I.D. Multimodular auxin response controls lateral root development in Arabidopsis. Plant Signal. Behav. 5, 580-582. (2010).

48. Su, L., Bassa, C., Audran, C., Mila, I., Cheniclet, C., Chevalier, C., Bouzayen, M., Roustan, J.P. \& Chervin C. The auxin SI-IAA17transcriptional repressor controls fruit size via the regulation of endoreduplication-related cell expansion. Plant Cell Physiol. 55, 1969-1976. (2014).

49. Su, Y.H., Liu, Y.B. \& Zhang, X.S. Auxin-cytokinin interaction regulates meristem development. Plant 4, 616-625. (2011).

50. Tang, L.P., Zhou, C., Wang, S.S., Yuan, J., Zhang, X.S. \& Su, Y.H. FUSCA3 interacting with LEAFY COTYLEDON2 controls lateral root formation through regulating YUCCA4 gene expression in Arabidopsis thaliana. New Phytol. 213, 1740-1754. (2017).

51. Vandesompele, J., Preter, K.D., Pattyn, F., Poppe, B., Roy, N.V., Paepe, A.D. \& Speleman, F. Accurate normalization of real-time quantitative RT-PCR data by geometric averaging of multiple internal control genes. Genome Biol. 3, 1-11. (2002).

52. Wightman, F., Schneider, E.A. \& Thimann, K.V. Hormonal factors controlling the initiation and development of lateral roots II. Effects of exogenous growth-factors on lateral root formation in pea roots. Plant 49, 304-314. (1980).

53. Yan, Y., Jing, X., Tang, H., Li, X., Gong, B. \& Shi, Q. Using transcriptome to discover a novel melatonininduced sodic alkaline stress resistant pathway in Solanum lycopersicum. Plant Cell Physiol. 60, 2051-2064. (2019).

54. Yuan, Y., Mei, L., Wu, M., Wei, W., Shan, W., Gong, Z., Zhang, Q., Yang, F., Yan, F., Zhang, Q., Luo, Y., Xu, X., Zhang, W., Miao, M., Lu, W., Li Z. \& Deng, W. SIARF10, an auxin response factor, is involved in 
chlorophyll and sugar accumulation during tomato fruit development. Exp. Bot. 69, 5507-5518. (2018).

55. Zha, M., Imran, M., Wang, Y., Xu, J., Ding, Y. \& Wang, S. Transcriptome analysis revealed the interaction among strigolactones, auxin, and cytokinin in controlling the shoot branching of rice. Plant Cell Rep. 38, 279-293. (2019).

\section{Figures}
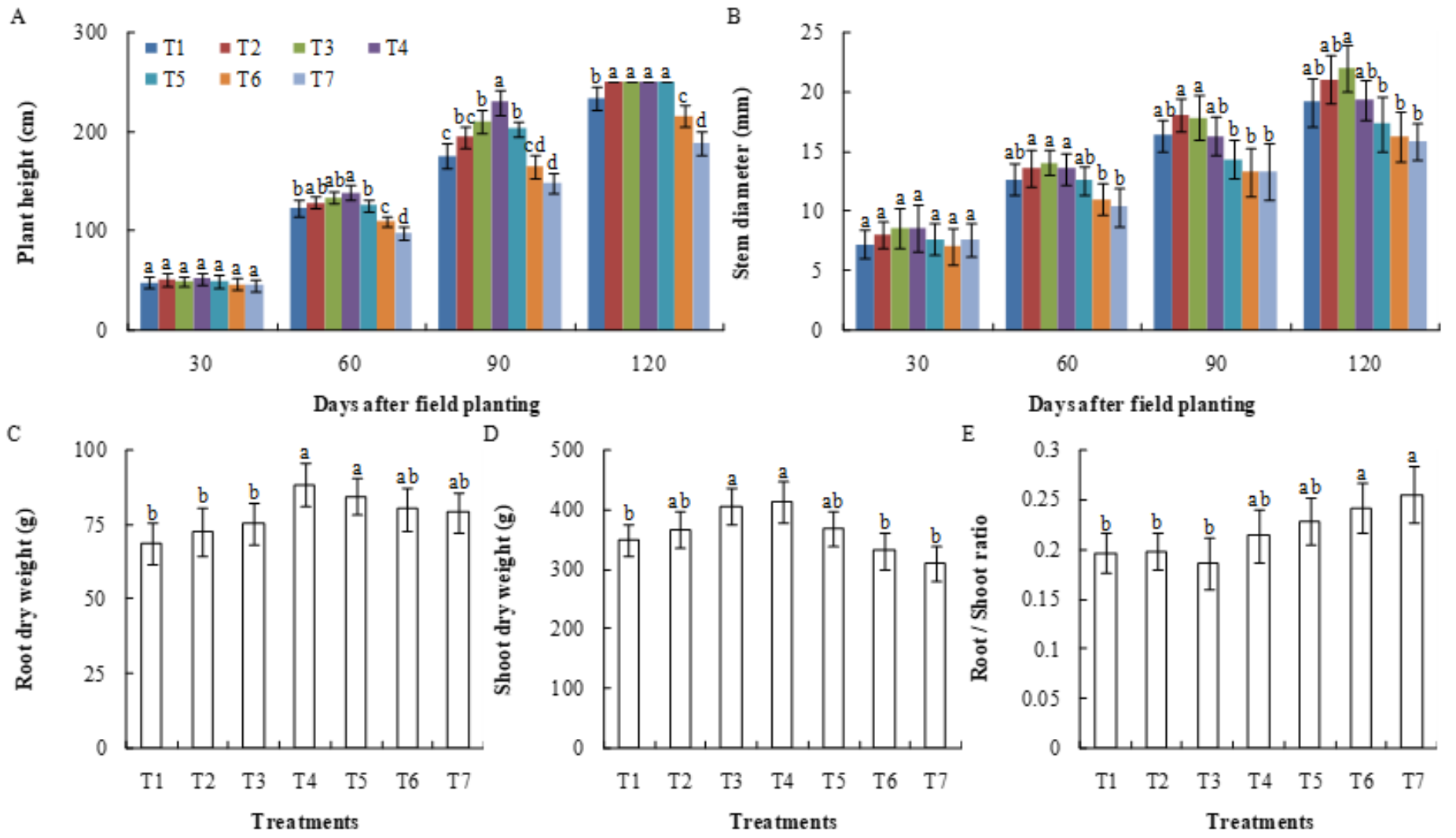

\section{Figure 1}

Influences of pruning length of LBs on plant growth. (A) Plant height and (B) stem diameter were measured at 30, 60, 90, $120 \mathrm{~d}$ after planting. (C) Root dry weight, (D) shoot dry weight and (E) root / shoot ratio were determined at $120 \mathrm{~d}$ after planting. Data are means of three different independent replicates $( \pm$ $\mathrm{SE})$. Different letters indicate significant difference at $\mathrm{P}<0.05$ between different treatments according to the Tukey's test. 

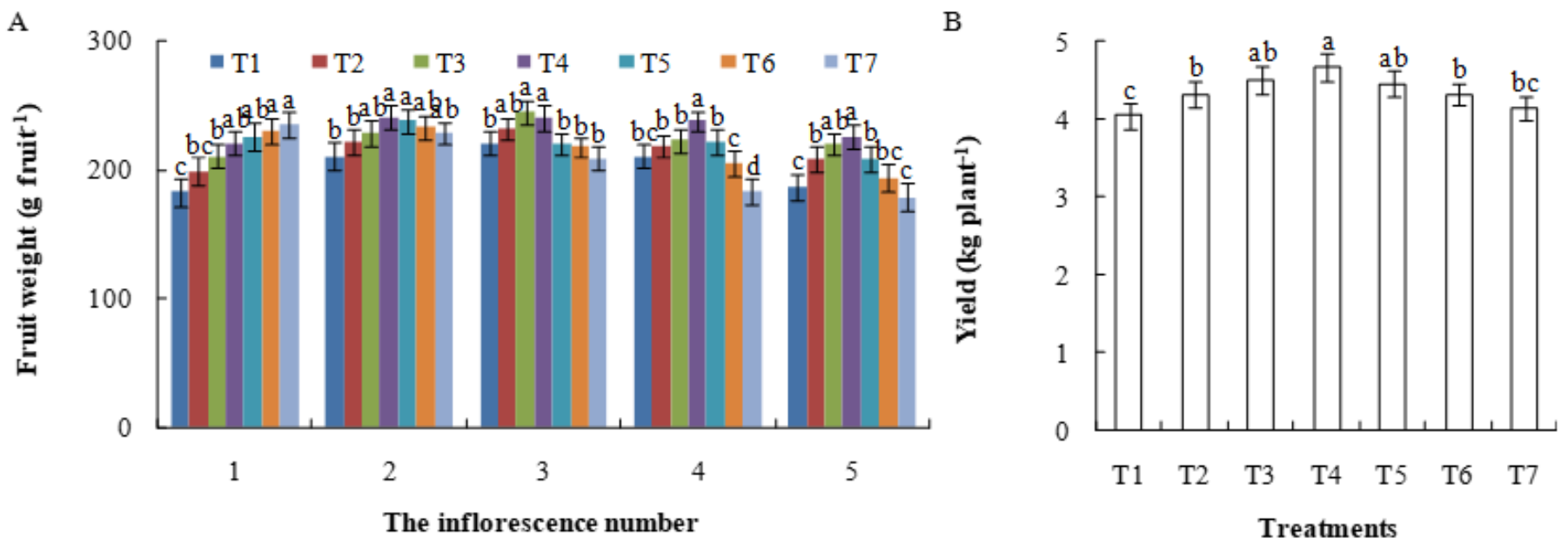

Figure 2

Influences of pruning length of LBs on yields. (A) Fruit weight of each inflorescence and (B) total yield were measured when the fruit ripening. Data are means of three different independent replicates $( \pm S E)$. Different letters indicate significant difference at $\mathrm{P}<0.05$ between different treatments according to the Tukey's test.

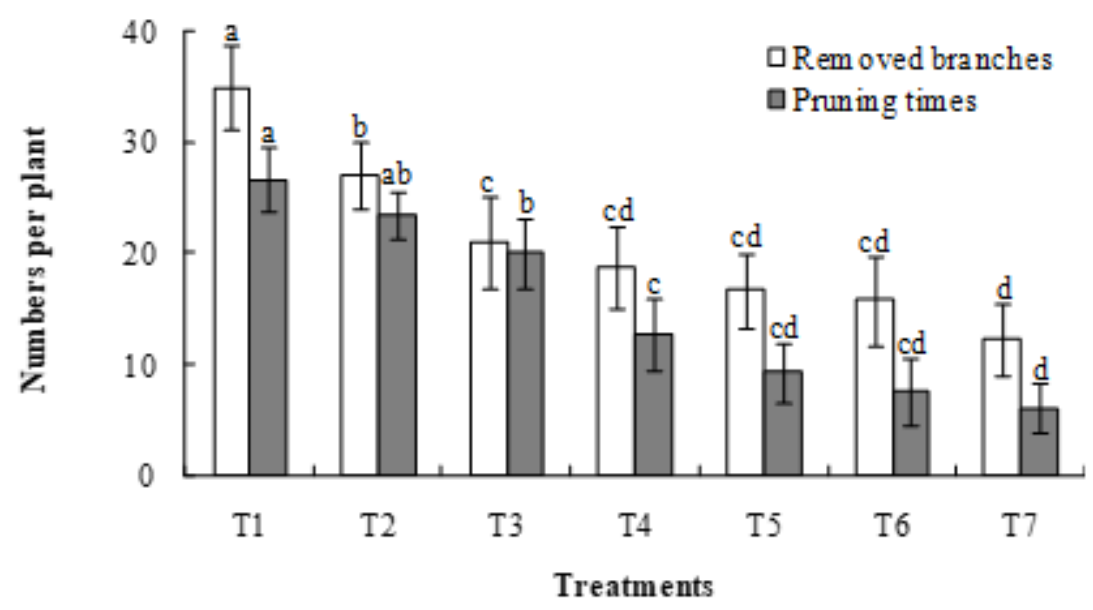

Figure 3

Influences of pruning length of LBs on labor costs. Numbers of removed branches and pruning times were recorded for labor costs evaluation. Data are means of three different independent replicates $( \pm S E)$. Different letters indicate significant difference at $\mathrm{P}<0.05$ between different treatments according to the Tukey's test. 

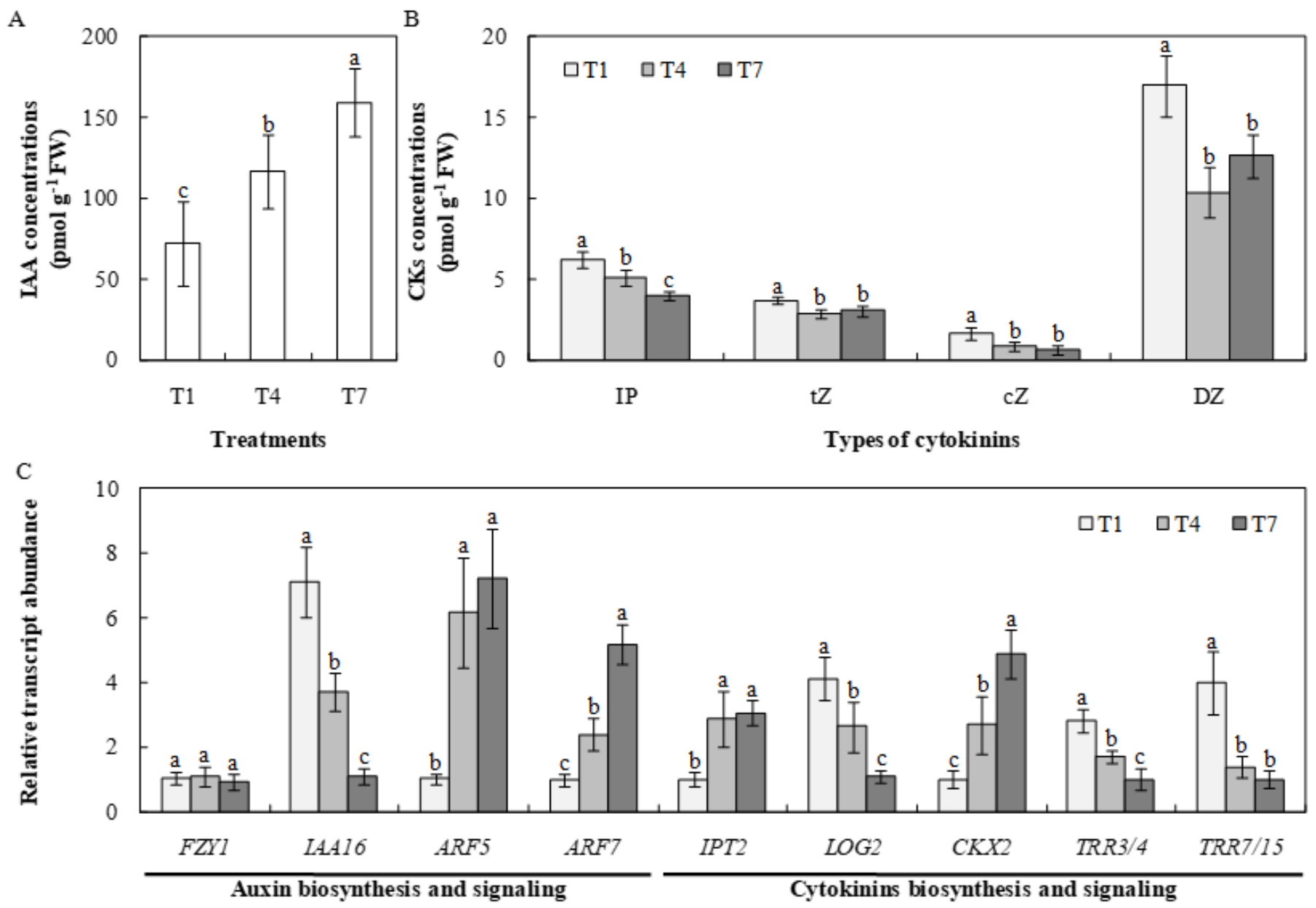

Figure 4

Influences of pruning length of LBs on auxin and CKs signaling in roots. (A) IAA concentrations, (B) CKs concentrations and (C) relative transcript abundance of auxin- and CKs-related genes were determined in tomato roots at $60 \mathrm{~d}$ after planting. Data are means of three different independent replicates $( \pm S E)$. Different letters indicate significant difference at $\mathrm{P}<0.05$ between different treatments according to the Tukey's test. 

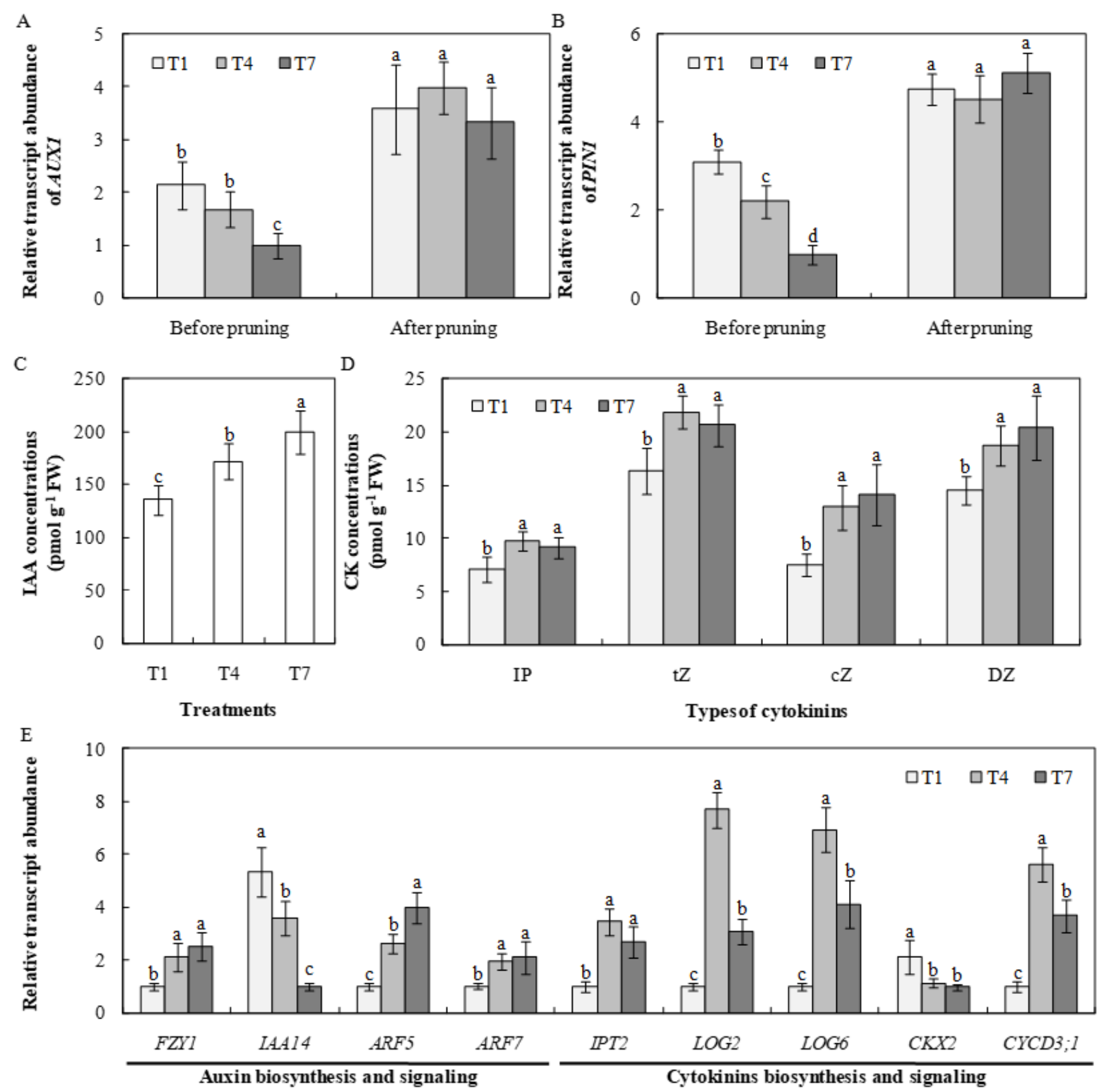

Figure 5

Influences of pruning length of LBs on auxin and CKs signaling in fruits. Relative transcript abundance of (A) AUX1 and (B) PIN1 were determined in the fruit stalks of the second inflorescence before and after pruning treatment. (C) IAA concentrations, (D) CKs concentrations and (C) relative transcript abundance of auxin- and CKs-related genes were determined in tomato ovaries at $5 \mathrm{~d}$ after anthesis. Data are means of three different independent replicates $( \pm S E)$. Different letters indicate significant difference at $P<0.05$ between different treatments according to the Tukey's test. 

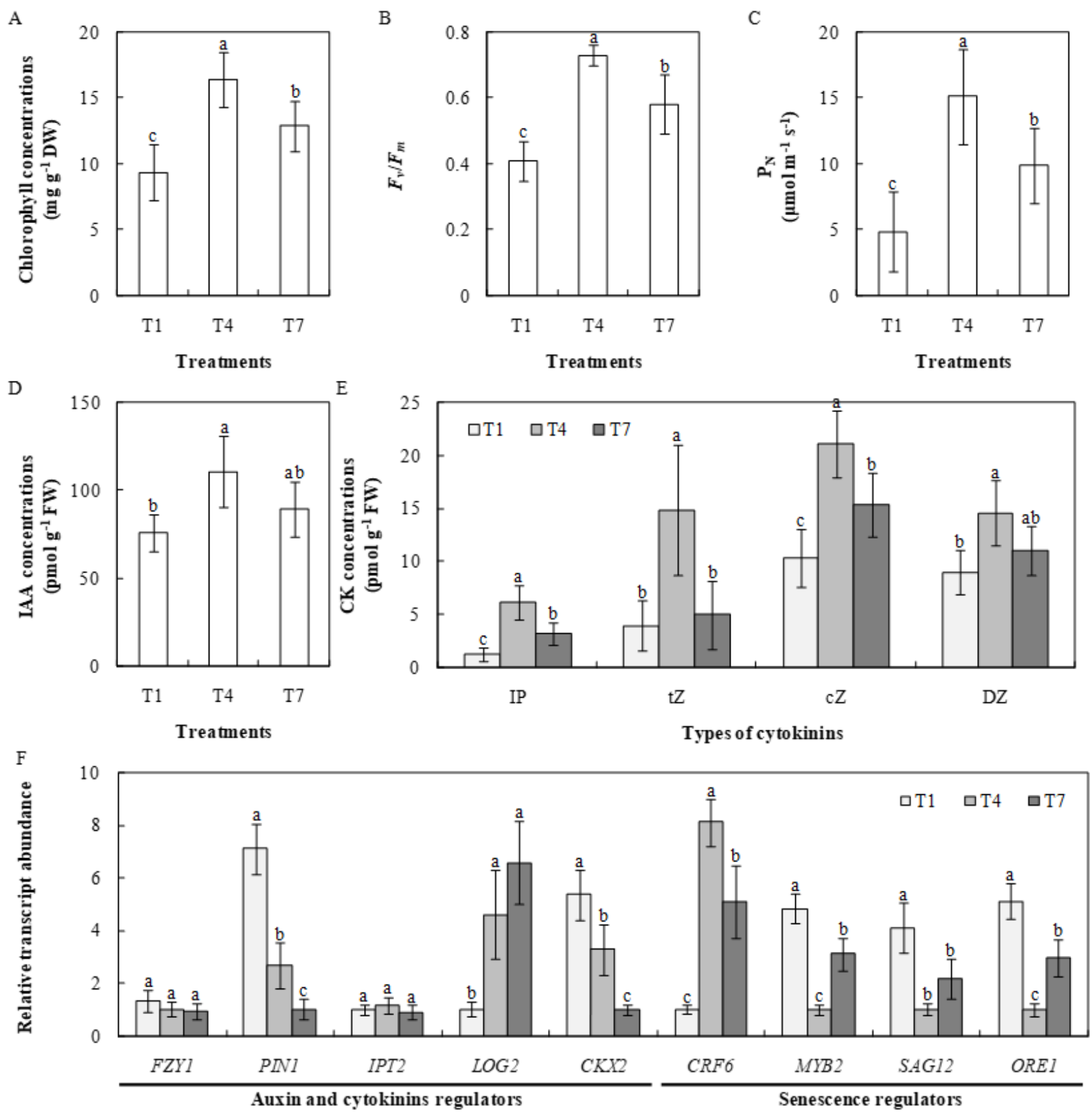

Figure 6

Influences of pruning length of LBs on auxin and CKs signaling in lower leaves. (A) Chlorophyll concentrations, (B) Fv/Fm, (C) PN, (D) IAA concentrations, (E) CKs concentrations and (F) relative transcript abundance of auxin- and CKs-related genes were determined in tomato lower leaves at $120 \mathrm{~d}$ after planting. Data are means of three different independent replicates $( \pm \mathrm{SE})$. Different letters indicate significant difference at $\mathrm{P}<0.05$ between different treatments according to the Tukey's test. 


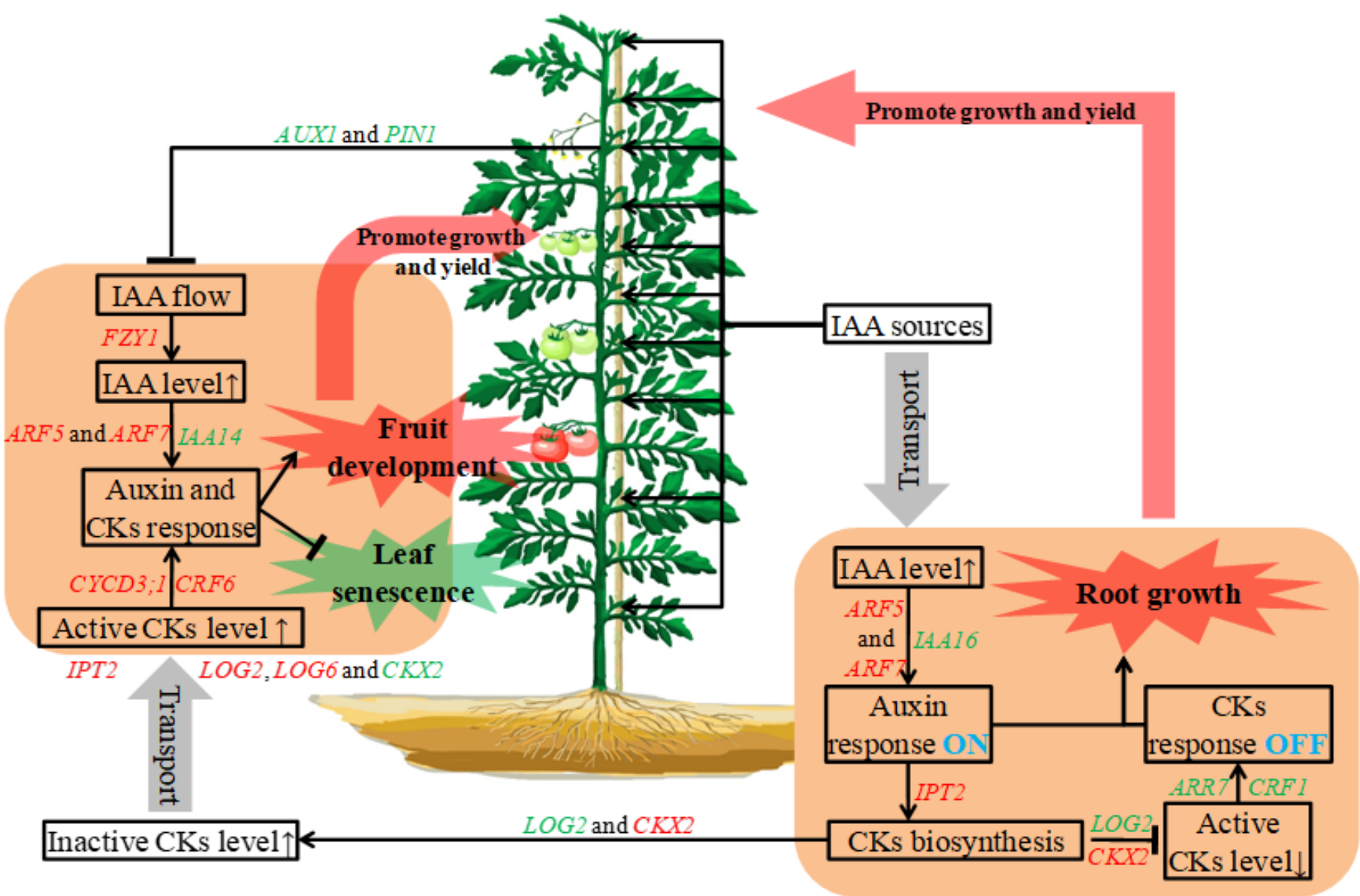

Figure 7

Proposed model for pruning length of LBs influences auxin and cytokinins homeostasis in relation to growth and yields in tomato plants. Promoted process is marked by red colour, and inhibited process is marked by green colour. 